\title{
PAPEL DA CURVA DE AGREGAÇÃO PLAQUETÁRIA NO CONTROLE DA ANTIAGREGAÇÃO NA PREVENÇÃO SECUNDÁRIA DO ACIDENTE VASCULAR CEREBRAL ISQUÊMICO
}

\author{
Patrícia Regina Piedade1, Rubens José Gagliardi², Ibsen Thadeo Damiani3, \\ Antonio Paulo Nassar Junior', Melissa Martins Fuzaro ${ }^{4}$, Wilson Luiz Sanvito ${ }^{5}$
}

\begin{abstract}
RESUMO - Introdução: O acidente vascular cerebral (AVC) é atualmente a primeira causa de morte no Brasil. 0 risco de recorrência de AVC é de aproximadamente $30 \%$ em cinco anos. Agentes antiagregantes plaquetários são frequentemente prescritos para a prevenção de recorrência do AVC, porém sem um parâmetro laboratorial de ajuste terapêutico. Objetivo: Determinar a aplicabilidade da curva de agregação plaquetária, no controle do antiagregante, na prevenção secundária do acidente vascular cerebral isquêmico (AVCi). Método: Estudo retrospectivo de janeiro de 1990 a janeiro de 2001; foram analisados 189 pacientes com diagnóstico de AVCi acompanhados durante este período com curva de agregação plaquetária. Foram excluídos os portadores de doenças cardioembólicas. Consideraram-se hipoagregados os doentes com agregação plaquetária ativada pelo ADP e/ou pela adrenalina menor ou igual a 50\%. Os doentes tiveram acompanhamento por 1 a 6 anos e a taxa de recorrência de AVCi foi comparada entre os normoagregados e os hipoagregados. Resultados: Nove doentes apresentaram novo AVCi no período do seguimento. Entre os hipoagregados ocorreu 2,6\% de recidiva e entre os normoagregados, $15,1 \%(0,03<0 R<0,69, p<0,05)$. A maioria dos doentes tiveram o novo episódio no segundo ano após o primeiro evento. Conclusão: A curva de agregação plaquetária mostrou ser um exame laboratorial eficiente como auxiliar para o controle da antiagregação na prevenção secundária do AVCi.
\end{abstract}

PALAVRAS-CHAVE: agregação plaquetária, acidente vascular cerebral, terapia antiagregante.

\begin{abstract}
Platelet aggregation test: application in the control of antiplatelet aggregation in the secondary prevention of stroke
\end{abstract}

ABSTRACT - Introduction: Stroke is the leading cause of death in Brazil. The risk of suffering a recurrent stroke is around $30 \%$ in 5 years. Antiplatelet therapy has been frequently used to prevent recurrent strokes without laboratory control. Objective: To determine the value of the platelet aggregation test in the control of antiplatelet aggregation in the secondary stroke prevention. Method: Retrospective study from January 1990 to January 2001 with 189 patients with stroke that were followed up during this period with platelet aggregation curve. Patients with cardioembolic disease were excluded. Low aggregation rates of platelet aggregation activated by ADP and/or epinephrine lower or equal to $50 \%$ were considered. Patients were followed up for a period from one to six years to verify differences in stroke recurrence. Results: Nine patients had had a recurrent stroke; $2,6 \%$ of the strokes occurred among the group with low aggregaton and $15,1 \%$ among the group with normal aggregation $(0,03<0 R<0,69, p<0,05)$. Most of the cases occurred in the second year after the first stroke. Conclusion: Platelet aggregation curve is a valid and a helpful laboratory test to therapeutic control of antiplatelet aggregation in secondary stroke prevention.

KEY WORDS: platelet aggregation curve, stroke, antiplatelet therapy.

O acidente vascular cerebral (AVC) é atualmente considerado a primeira causa de incapacidade motora, sensitiva e cognitiva no mundo ${ }^{1}$, a primeira causa de morte no Brasil $^{2}$ e a segunda causa de morte na maioria dos países desenvolvidos ${ }^{3}$. O risco de recorrência de AVC situa-se entre 15 e $30 \%$ em cinco $\operatorname{anos}^{1,4}$, o que corresponde a uma chance nove vezes maior que a da população geral ${ }^{1}$. O novo episódio de doença cerebrovascular geralmente tem maior gravidade para o paciente e implica em significativo ônus para o sistema de saúde ${ }^{5}$. A demência, o declínio da função cognitiva e a incapacidade motora

\footnotetext{
Disciplina de Neurologia e Liga da Aterosclerose, Faculdade de Ciências Médicas da Santa Casa de São Paulo, São Paulo SP, Brasil ${ }^{1}$ Residente de Neurologia; ${ }^{2}$ Professor Adjunto, responsável pelo Setor de Doenças Cerebrovasculares; ${ }^{3}$ Professor Instrutor; ${ }^{4}$ Acadêmico de Medicina; ${ }^{5}$ Professor Titular.
}

Recebido 2 Janeiro 2003, recebido na forma final 28 Março 2003. Aceito 15 Abril 2003.

Dr. Rubens José Gagliardi - Disciplina de Neurologia - Departamento de Medicina - Santa Casa de São Paulo - Rua Dr. Cezário Motta Jr 112 - 01221-020 São Paulo SP - Brasil. E-mail: neurologiasantacasasp.org.br 
são complicações frequentes e temidas após a recorrência do AVC, com importantes implicações sócioeconômicas ${ }^{6}$. Nos EUA, $20 \%$ do episódios de AVC ao ano são recorrentes ${ }^{1}$. As principais causas de AVC são eventos trombóticos ou embólicos ${ }^{7}$. As plaquetas exercem um papel fundamental na hemostasia normal e passam a ter também uma participação importante na formação de trombos e êmbolos ${ }^{8,9}$.

Após uma lesão vascular, as plaquetas encontram constituintes da matriz extracelular, especialmente o colágeno, e são ativadas. Em contato com esta, as plaquetas reagem de três formas: 1 . aderência à matriz extracelular; 2 . secreção do conteúdo de seus grânulos; 3. agregação a outras plaquetas. A aderência plaquetária à matriz extracelular decorre principalmente da interação entre o fator de von Willebrand (fvW) e o colágeno. O fvW atua como uma ponte de aderência entre o colágeno e o receptor de glicoproteína lb plaquetário. A secreção do conteúdo granular é desencadeada pela ligação de agonistas aos receptores de superfície das plaquetas. A liberação desse conteúdo é importante porque existem elementos, como o difosfato de adenosina (ADP), que favorecem a agregação plaquetária e outros, como o cálcio, que têm papel fundamental na cascata de coagulação. A agregação plaquetária, consequente ao ADP (contido no grânulo plaquetário a) e ao tromboxano $A_{2}$ (sintetizado com a ativação plaquetária) leva à formação do tampão hemostático primário. Com a ativação da cascata da coagulação, a trombina gerada liga-se à superfície plaquetária, resultando em mais agregação. O fibrinogênio também é um co-fator na agregação plaquetária, associando-se às plaquetas através dos receptores de glicoproteína Ilb/IIla. A associação entre aterosclerose e trombose ocorre porque as placas ateroscleróticas ulceradas levam à formação de trombos pela exposição da matriz extracelular subendotelial e por serem fontes de turbulência do fluxo sanguíneo local ${ }^{10}$.

As medidas antiplaquetárias oferecem benefício no tratamento da doença tromboembólica arterial por bloqueio ao depósito de plaquetas na superfície de uma placa arterosclerótica rota ${ }^{11}$. Os agentes antiagregantes plaquetários previnem novos acidentes vasculares cerebrais ${ }^{11}$, sendo as drogas de primeira escolha para esse fim $^{12}$. 0 ácido acetilsalicílico (AAS) é o agente antiplaquetário mais extensamente estudado, o mais barato e o mais usado ${ }^{7}$. Ticlopidina, clopidogrel, dipiridamol e antagonistas de receptor de glicoproteína Ilb/IIla são outros antiagregantes utilizados ${ }^{11}$.

O AAS exerce seu efeito antitrombótico pela inibição irreversível da ciclo-oxigenase plaquetária, levando à redução da síntese de TXA ${ }_{2}$, comprometendo a agregação plaquetária. $\mathrm{O}$ dipiridamol inibe a fosfodiesterase, alterando a função plaquetária. A ticlopidina e o clopidogrel inibem a ligação do ADP ao seu receptor plaquetário. Agem também modificando a resposta plaquetária a outros agonistas além do fibrinogênio. A agregação plaquetária também é prevenida quando antagonistas agem no receptor GPIIb/IIla ${ }^{13}$. Essas drogas, com exceção dos antagonistas da GPIlb/Illa, são frequentemente prescritas para prevenção secundária de AVC, porém sem um parâmetro laboratorial de ajuste terapêutico.

$O$ estudo da agregação plaquetária através da curva de agregação (CAP) é útil na avaliação da função das plaquetas, pela exploração de diferentes vias de ativação plaquetária in vitro. Este estudo pode ter um papel importante ao auxiliar o controle clínico para minimizar a recidiva do AVC. O método é baseado na medida da formação de agregados de plaquetas após sua exposição a um agente agregante. Esta medida é realizada em um agregômetro, que é um tipo de espectrofômetro capaz de medir a variação da transmissão da luz através de uma suspensão de plaquetas, quando estas se agregam na presença de agonistas. Há vários agonistas usados na prática: o colágeno, o ADP, a adrenalina, o ácido araquidônico e a trombina. $O$ resultado do teste é habitualmente expresso em porcentagem da agregação, que traduz a quantidade de transmissão de luz e portanto da formação de agregados ${ }^{14}$.

Este estudo tem por objetivo determinar o papel da curva de agregação plaquetária, no controle do agente antiagregante, na prevenção secundária do acidente vascular cerebral isquêmico (AVCi).

\section{MÉTODO}

Estudo retrospectivo, do período de janeiro de 1990 a janeiro de 2001, com doentes acompanhados no ambulatório da Liga de Aterosclerose do Serviço de Neurologia da Faculdade de Ciências Médicas da Santa Casa de São Paulo.

Os critérios de inclusão foram: doentes com AVCi aterotrombótico que mantiveram o uso regular de algum antiagregante plaquetário, e que fizeram acompanhamento laboratorial com a curva de agregação plaquetária, com intervalos de um a seis meses, concomitantemente com avaliação clínico-neurológica. A CAP foi realizada sob a indução de ADP e adrenalina através do agregômetro Net Lab digital platelet. Foram excluídos os doentes que não tomaram regularmente o AG prescrito, os que não mantiveram adequadamente o tratamento da doença de base (fator de risco), os que por qualquer motivo não retornaram às consultas antes do término do período de avaliação e os portadores de doenças cardioembólicas. Foram selecionados 189 doentes. 
Foram considerados hipoagregados os doentes com agregação após a indução por ADP e/ou adrenalina menor ou igual a $50 \%$ e normoagregados aqueles, que com o mesmo método, mostraram valores maiores de $50 \%$.

Os pacientes foram acompanhados por um período de um a seis anos, procurando verificar se havia diferença na recorrência de AVCi entre os grupos de hipoagregados e de normoagregados. Foi utilizado o método estatístico do qui-quadrado para comparação entre os grupos.

O tipo e a dose do antiagregante prescrito, não foram motivo de estudo, pois o presente trabalho visou avaliar o índice de agregação através de um procedimento laboratorial (curva) e não o fármaco ou a dose utilizada.

Todos os doentes nos dois grupos receberam os tratamentos clínico e neurológico rotineiros, necessários a cada caso.

\section{RESULTADOS}

Dos 189 pacientes estudados, 95 (50,3\%) eram do sexo feminino e $94(49,7 \%)$ do sexo masculino. A média de idade foi 57,7 anos.

Pela avaliação da curva de agregação plaquetária, observou-se que 156 (83\%) doentes estavam hipoagregados e 33 (17\%) estavam normoagregados. No grupo de hipoagregados, a média de idade foi de 62,8 anos, enquanto no grupo de normoagregados, a idade média foi 56,6 anos. Quanto à distribuição por sexo, no grupo de normoagregados houve predominância feminina. (Tabela 1).

Nove pacientes $(4,8 \%)$ sofreram um novo episódio de AVC durante o período de acompanhamento ambulatorial. A média de idade foi 52,3 anos. A recorrência de AVC foi significativamente maior no grupo de normoagregados em comparação aos hipoagregados (Tabela 2).

O período em que houve maior recorrência de AVC foi no segundo ano após o ícto AVC (Tabela 3).

\section{DISCUSSÃO}

No presente estudo, encontrou-se uma recorrência maior de AVC em pacientes que estavam normoagregados em comparação aos hipoagregados, avaliados através da CAP. Tal fato sugere um importante papel do acompanhamento laboratorial da agregação plaquetária para o manejo terapêutico dos antiagregantes plaquetários na prevenção secundária do AVCi. Não foi encontrado estudo semelhante na literatura. Os pacientes foram acompanhados com curva de agregação plaquetária induzidas por agentes agregantes primários, ADP e adrenalina, que são capazes de iniciar a agregação plaquetária diretamente por mecanismos independentes da indução de produção de prostaglandinas ou da liberação de grânulos plaquetários contendo $A D P^{10}$. É um método que tem se mostrado eficaz para o controle da agregação ${ }^{10,15}$.

O índice laboratorial menor ou igual a $50 \%$ após a indução com ADP e/ou adrenalina empregado neste estudo para definir um determinado resultado como

Tabela 1. Distribuição por sexo de acordo com a agregação plaquetária.

\begin{tabular}{lccc}
\hline $\begin{array}{l}\text { Agregação } \\
\text { plaquetária }\end{array}$ & Feminino(\%) & Masculino(\%) & Total \\
\hline Normoagregados & $22(66,7)$ & $11(33,3)$ & 33 \\
Hipoagregados & $73(46,8)$ & $83(53,2)$ & 156 \\
Total & $95(50,3)$ & $94(49,2)$ & 189 \\
\hline
\end{tabular}

$O R=0,44(p<0,05)$

Tabela 2. Evolução (recorrência ou não de AVCi) segundo a agregação plaquetária.

\begin{tabular}{lccc}
\hline $\begin{array}{l}\text { Agregação } \\
\text { plaquetária }\end{array}$ & $\begin{array}{c}\text { Com AVCi } \\
\text { recorrente(\%) }\end{array}$ & $\begin{array}{c}\text { Sem AVCi } \\
\text { recorrente(\%) }\end{array}$ & Total \\
\hline Normoagregados & $5(15,1)$ & $28(84,9)$ & 33 \\
Hipoagregados & $4(2,6)$ & $152(97,4)$ & 156 \\
Total & $9(4,8)$ & $180(95,2)$ & 189 \\
\hline
\end{tabular}

$O R=0,15(p<0,05)$

Tabela 3. Recorrência de AVCi segundo a agregação plaquetária, por tempo de seguimento.

\begin{tabular}{cccccc}
\hline \multirow{2}{*}{$\begin{array}{c}\text { Tempo de } \\
\text { seguimento } \\
\text { (em anos) }\end{array}$} & Com recorrência & Sem recorrência & Com recorrência & Sem recorrência \\
\hline 1 & 1 & 15 & 0 & 78 & 94 \\
2 & 2 & 5 & 2 & 23 & 32 \\
3 & 1 & 2 & 0 & 18 & 21 \\
4 & 1 & 3 & 1 & 14 & 19 \\
5 & 0 & 1 & 0 & 6 & 7 \\
6 ou mais & 0 & 2 & 1 & 13 & 16 \\
Total & 5 & 28 & 4 & 152 & 189 \\
\hline
\end{tabular}


hipoagregado tem sido citado na literatura como parâmetro confiável ${ }^{15}$. No presente estudo não foi analisada a possível influência dos clássicos fatores de risco ateroscleróticos, pois o objetivo primário foi verificar a aplicabilidade da CAP como auxiliar para o controle da antiagregação, independentemente do tipo do doente.

A prevalência da faixa etária acima de 60 anos e a média de idade dos pacientes que tiveram um AVC foi a mesma encontrada em outros estudos ${ }^{1,5}$. Estudos epidemiológicos indicam que o risco de recorrência é maior durante o primeiro ano após o AVC inicial (cerca de 13\%) 4,16 , correspondendo secundariamente a um risco anual de aproximadamente $4 \%$ em pacientes não tratados ${ }^{1}$. Nos pacientes acompanhados neste estudo, a maior recorrência de AVCi esteve presente no segundo ano após o episódio e a taxa total de recorrência em todos os pacientes acompanhados de 1 a 6 ou mais anos foi de $4,8 \%$. Este índice é relativamente baixo, fato possivelmente explicado pela maioria dos pacientes estarem hipoagregados ( $83 \%$ ) e em controle ambulatorial dos fatores de risco de aterosclerose.

O papel dos diferentes agentes antiagregantes não foi objeto do presente estudo, não havendo comparação entre os pacientes que faziam uso dos diferentes fármacos disponíveis. A maioria dos nossos doentes estavam em uso de AAS. Este e outros agentes antiagregantes são protetores na maioria dos pacientes com risco de desenvolverem eventos vasculares trombóticos ${ }^{11}$. No entanto, parecem não influenciar a gravidade do AVC recorrente quando este ocorre ${ }^{17}$.

Quanto ao custo, foi demonstrado que o tratamento diário, em dólares, com AAS é de US $\$ 0,06$, o que leva a um despesa anual de US\$24,09. A associação AAS e dipiridamol custa US\$2,95 diários e US\$ 1076,75 anuais. Para o tratamento com clopidogrel, os valores são US\$ 3,01 e US\$1098,68, respectivamente ${ }^{1}$. Segundo tabela da Associação Médica Brasileira, a curva de agregação plaquetária custa aproximadamente US\$12,60, o que sugere que o uso do AAS em doses efetivas, quando não houver contra-indicação clínica, supervisionado pela CAP para se obter um grau de hipoagregação efetivo, e a consequente prevenção secundária de AVC traz segurança e economia.

Dos quatro pacientes hipoagregados $(2,6 \%)$ que tiveram recorrência de AVC neste estudo, observase que dois pacientes apresentaram acidente vascular cerebral isquêmico transitório, um paciente apresentava diabetes mellitus de difícil controle e o outro era do sexo feminino, jovem (30 anos), com hipoagregação de $5 \%$ sob ADP porém com $68 \%$ sob adrenalina. Dessa forma, apesar do resultado estatistica- mente significativo já apresentado quanto à curva de agregação plaquetária, seria interessante particularizar os fatores de risco destes pacientes, aprofundando o diagnóstico etiológico para determinar se, nesses casos, somente a hipoagregação seria a melhor terapêutica para prevenção secundária de AVC. Este estudo mostra também a importância da sua continuidade com a análise da curva de agregação plaquetária combinada a diferentes agentes antiagreagantes e especificada para subgrupos com diferentes fatores de risco e diferentes tipos de AVCi, possibilitando a verificação de possível interação destes elementos, para uma adequada supervisão de dosagem e posologias específicas necessárias para atingir a hipoagregação esperada para cada caso específico, para evitar a recorrência do AVC.

\section{CONCLUSÃO}

A curva de agregação plaquetária mostrou ser, neste estudo, um método laboratorial eficiente como auxiliar para o controle da antiagregação na prevenção secundária do ACVi. Este estudo sugere que a curva de agregação plaquetária possa ser um método de controle da antiagregação plaquetária de auxílio na prevenção secundária do AVCi, sendo necessários estudos adicionais para melhor avaliação.

\section{REFERÊNCIAS}

1. Shah H, Gondek K. Aspirin plus extended - release dipyridamole or clopidogrel compared with aspirin monotherapy for the prevetion of recurret ischemic stroke: a cost effectiveness analysis. Clin Ther 2000;22:363-370.

2. Lessa I. Epidemiologia das doenças cerebrovasculares no Brasil. Rev Soc Cardiol Est SP 1999;4:509-518

3. Murray CJ, Lopez AD. Mortality by cause for eight regions of the world: Global Burden of Disease Study. Lancet 1997; 49:1269-1276.

4. Hankey GJ, Jamrozik K, Broadhurst RJ. Long-term risk of first recurrent stroke in the Perth Community Stroke Study. Stroke 1998;29:2491-2500.

5. Samsa GP, Bian J, Lipscomb J, Matchar DB. Epidemiology of recurrent cerebral infarction: a medicare claims-based comparison of first recurrent strokes on 2-year survival and cost. Stroke 1999;30:338-349.

6. Inzitari D, Di Carlo A, Pracucci G. Incidence and determinants of poststroke dementia as defined by an informant interview method in a hospital-based stroke registry. Stroke 1998;29:2087-2093.

7. Bronner LL, Kanter DS, Manson JE. Primary prevention of stroke. New Engl J Med 1995; 333:1392-1400.

8. Packam MA. Roles of platelets in thrombosis and hemostasis. Can J Physiol Pharmacol 1994;72:278-284.

9. Harker LA. Platelets and vascular thrombosis. N Engl J Med 1994;330:1006-1007

10. Mitchell RN, Cotran RS. Distúrbios hemodinâmicos, trombose e choque. In Cotran RS, Kumar V, Robbins S.L. (EDS) - Robbins patologia estrutural e funcional. 6.Ed. Rio de Janeiro: Guanabara-Koogan, 2000:107-116.

11. Antithrombotic Trialists' Collaboration. Collaborative meta-analysis of randomised trials of antiplatelet therapy for prevention of death, myocardial infarction, and stroke in high risk patients. BMJ 2002;324:71-86.

12. Lees KR, Bath PM, Naylor AR. ABC of arterial and venous disease. Secondary prevention of transient ischaemic attack and stroke. BM] 2000;320:991-994.

13. Lee RG, Foerster J, Lukens J, Paraskevas F, Greer JP, Rodgers GM. Wintrobes clinical hematology. 10.Ed. Baltimore: Lippincott Williams \& Williams, 1999:1806-1808

14. Wu KK, Thiagarajan $\mathrm{R}$. Role of endothelium in trombosis and hemosthasis. Ann Rev Med 1996;47:315-331.

15. Kawasaki T, Ozeki Y, Ygawa, T, Kambasi J. Increased platelet sensivity to collagen in individuals resistant to low-dose aspirin. Stroke 1999;31:591-595.

16. Burn J, Dennis MS, Bamford J, Sandercock PAG, Wade D, Warlow CP. Long-term risk of recurrent stroke after a first-ever stroke: The Oxfordshire Community Stroke Project. Stroke 1994;25:333-337.

17. Sivenius J, Cunha L, Dienner, HC. et al. Antiplatelet treatment does not reduce the severity of subsequent stroke. Neurology 1999;53:825-829. 